\title{
A Novel Method for VHF Signal Modulation Classification Based on Algorithm of First-Order Cyclic Moment
}

\author{
F.Q. Yang \\ School of Electronic and Information Engineering \\ Foshan University \\ Foshan, China \\ Z. $\mathrm{Li}$ \\ State Key Laboratory of Integrated Service Networks \\ Xidian University \\ Xi'an, China
}

\begin{abstract}
In view of the problem of modulation recognition algorithm existing about low recognition rate under environment condition of low SNR, the algorithm of first-order cyclic moment are first presented used in band signal of VHF modulation classification recognition in this paper and the recognition rate increased significantly. The first-order cycle frequencies at which estimated first-order cyclic moment magnitudes exceed the cutoff value were selected as candidate cycle frequencies based on estimation first-order cyclic moment at first-order cycle frequency series, then decided to choose the number of candidate cycle frequency by a cyclostationarity test to realize signal modulation classification recognition, the classification recognition rate can reach more than $98 \%$ under condition of SNR is greater than $-6 \mathrm{~dB}$.
\end{abstract}

Keywords-first-order cycle frequency; first-order cyclic moment magnitudes; cut-off value; band of VHF; modulation classification

\section{INTRODUCTION}

Band of VHF is widely used in military and civil communication. For example, modulation system mainly included AM, ASK, FM, FSK, DSB, SSB, and single carrier linear digital modulation (SCLD) (e.g. PSK, QAM), as well as cyclic prefix single carrier linear digital modulation (CP-SCLD) et al are used in the industries such as electric power, water conservancy, weather, petroleum, forestry, exploration, coal, railway et al, which reach the digital signals transmission of communication, remote control, telemetering, remote sensing, local alarm system. Therefore, it is of significance that the monitoring of communication signals, signal confirmation, interference recognition, electronic rescue and analysis of military threat can be reached by the modulation recognition of this band of VHF[1-2].

The process of modulation recognition includes three parts (signal preprocessing, feature extraction, classification and identification). In the signal preprocessing, following-up treatment is provided the appropriate data which mainly includes frequency inverter, synthetic and quadrature component decomposition, estimation of the carrier frequency

\author{
Z.L. Luo \\ Huizhou University \\ Huizhou, China
}

\author{
B.J. Hao \\ State Key Laboratory of Integrated Service Networks \\ Xidian University \\ Xi'an, China
}

and the signal rate, and multipath channel equalization et al. Feature extraction means the information which is useful in modulation recognition is extracted from input signal sequence so as to obtain the feature that can reflect the difference most. Classification and identification mainly concerns about the judegment of affiliation of signal modulation types[3-4]. Meanwhile, the current main method of feature extraction is repective extraction in eigenvalue of the instant frequency, eigenvalue of time domain, analysis of high order origin moment, high-order cumulants, wavelet transform, spectral correlation, cyclic spectral correlation, power spectrum, planisphere, signal kurtosis et al[5-8]. This method reaches the great the recognition effect. But with the increasingly complex signal environment, it is a huge challenge in how to make innovation in the basis of current recognition technology, and promote classification ability under the situation of the serious interference of white Gaussian noise and phase-frequency distortion and time delay et al[9]. Therefore, stationary characteristic of first-order cyclic is applied and the algorithm of mean value of the first-order cyclic in this paper to be used in the modulation classification recognition of band signal of VHF. The information about signal carrier frequency, signal bandwidth et al are not needed in this algorithm and this algorithm can reach the higher classification ability under the condition of low SNR.

\section{THE AlgORITHM OF FIRST-ORDER CYCLIC MEAN VALUE AND RECOGNITION STEPS}

\section{A. The Algorithm of First-Order Cyclic Mean Value}

The recognition parameter of first-order cyclic stationary applied in this paper mainly includes: first-order cyclic series(CFS), first-order cyclic mean value (CM) and the absolute value of first-order cyclic mean value[10-12]. the algorithm used in this paper consists of the following:

1) The magnitude of the first-order $\mathrm{CM}$ of the normalized signal is estimated at candidate CFs $\alpha^{\prime}$, over a range corresponding to the bandwidth normalized to the sampling 
rate, and based on a $k$ sample observation interval. A cut-off value $V_{C O}$ is set, and candidate $\mathrm{CFs}$ which correspond to a $\mathrm{CM}$ magnitude above or equal to $V_{C O}$ are selected for testing in the next step. The notation $S N R$ co will be subsequently used for this SNR. It is noteworthy that for SNRs well above SNR co, peaks corresponding to all CFs will be tested, whereas for SNRs well below SNR co, these will lie below the cut-off value $V_{C O}$, and be missed.

2) Checking whether or not the candidate CFs selected in (1) of the algorithm are indeed CFs. A first-order CM-based statistic is estimated at each candidate $\mathrm{CF}$, and compared against a threshold. If the estimated statistic at a candidate $\mathrm{CF}$ exceeds the threshold, then the candidate is decided to be a CF. The number of CFs is finally employed to make a decision on the signal detection and classification.

\section{B. Recognition Steps}

Based on the recognition algorithm above which classify and recognize the brand signal of $\mathrm{VHF}$, the classification recognition steps:

Step 1: Filtering out-of-band noise, treatment of downconversion and standard digital processing in un-recognized identification signal;

Step 2 : Estimation of the first-order CM magnitude at candidate CFs over the range corresponding to the signal bandwidth normalized to sampling frequency;

Step 3: Selection of candidate CFs at which estimated firstorder CM magnitudes exceed the cut-off value $V_{C O}$;

Step 4: Determination of the number of CFs by applying a cyclostationarity test to selected candidate $\mathrm{CFs}$;

Step 5: Classification and recognition of brand signal of VHF (including AM, ASK, MASK, FM, 2FSK, MFSK, DSB, SSB, SCLD, CP-SCLD et al) based on the candidate of the number first-order cyclic frequency.

After that, in the receiving end, the receipt signal will be $\mathrm{AM}$ and ASK if first-order cyclic frequency is detected. If two first-order cyclic frequency is detected, the receiving signal will be 2 FSK. If the first-order cyclic frequency is detected as number $M$ in $\left(2^{m-1}+1,2^{m}\right)$, the receipt signal will be MFSK $\left(\mathrm{M}=2^{\mathrm{M}}, \mathrm{M} \geq 4\right)$. If no first-order cyclic frequency is detected, the receipt signal will be DSB, SSB, SCLD, CP-SCLD.

\section{THE THEORETICAL ANALYSIS OF RECOGNITION METHOD PERFORMANCE}

In the algorithm discussed above, the method of measuring stationarity is applied in seeking the number and frequency of first-order cyclic. Meanwhile, these frequency are regarded as the candidate of first-order cyclic frequency and their related absolute value of first-order cyclic mean value are bigger than cutoff value $V_{C O}$. Based on the theory, when the SNR is lower than SNR co, mean value of the first-order cyclic absolute value which is long data sequence is lower the cutoff value $V_{C O}$. So in the algorithm, it is impossible that the related firstorder cyclic frequency and the classification ability of signal is zero by the method of measuring stationarity. When the SNR is bigger than $S N R$ CO, all first-order frequency can be detected, which the classification ability of AM or ASK signal is equal to the probability by the measurement of stationarity (also called the probability by the measurement of first-order cyclic frequency):

$$
P_{A M / A S K}=P^{(1)}
$$

The probability of correct classification for 2FSK and MFSK signals is given by the probability of detecting two and between $2^{m-1}+1$ and $2^{m} \mathrm{CFs}$, respectively:

$$
\begin{gathered}
P_{2 F S K / M F S K}=\prod_{N_{o}=1}^{M} P^{\left(N_{o}\right)}+\sum_{m=1}^{M / 2-1, M \geq 4} R_{m} \\
R_{m}=\sum_{\substack{N_{m} \geq 1, \cdots, N_{m} \geq 1 \\
N_{m} \succ \cdots N_{1}}}^{M} \prod_{N_{m+1} \in S_{\left(N_{1}, \cdots N_{m}\right)}} P^{\left(N_{m+1}\right)} . \\
\left(1-P^{\left(N_{m}\right)}\right) \cdots\left(1-P^{\left(N_{1}\right)}\right) \\
S_{\left(N_{1}, \cdots, N_{m}\right)}=\left\{\begin{array}{l}
N: 1 \leq N \leq M, \\
N \neq N_{1}, \cdots, N_{m}
\end{array}\right\}
\end{gathered}
$$

where $P^{(N)}$ is the probability of detecting the Nth CF, when SSB, DSB, SCLD, or CP-SCLD signals are present at the receive-side, no candidate $\mathrm{CFs}$ are selected, as such, no firstorder CFs are detected, and the probability of detecting noise or such signals equals one.

\section{The RESUlt AND ANALYSIS OF SimULATION}

M-FSK, $M=2,4$, and 8, and AM signals with a single-sided bandwidth of $3 \mathrm{kHz}$ and unit power are simulated. The observation interval available at the receive-side is 1 second, which is equivalent to $1500 \mathrm{~B}$ 2FSK symbols, 750B 4FSK symbols, and 375 8FSK symbols, and the frequency deviation equals $\Delta f=2400 H_{Z}$. For the AM signal, the modulating signal $m(t)$ is obtained by low-pass filtering a sequence of zero-mean Gaussian random numbers, with unit variance. The modulation index $\mu_{A}$ is set to 0.3. The time delay $t_{0}=0.6 T$ and $t_{0}=10 f_{S}^{-1}$ for the MFSK and AM signals, respectively. The carrier phase $\theta$ is uniformly distributed over $(-\pi \pi)$, The received signals are sampled at rate $f_{S}=48 k H_{Z}$, and set the cutoff value $V_{C O}=0.05$, the number of simulation experiments are 1000 times.

In the same time slot of observation and the condition of different SNR, signal of AM, 2FSK, 4FSK, SFSK et al in the brand of VHF are proceed modulation classification and 
recognition. The result of simulation can be seen in the Figure 1 :

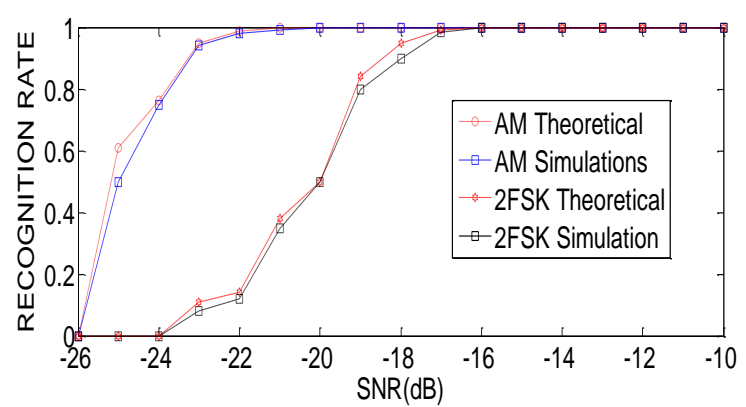

(a)

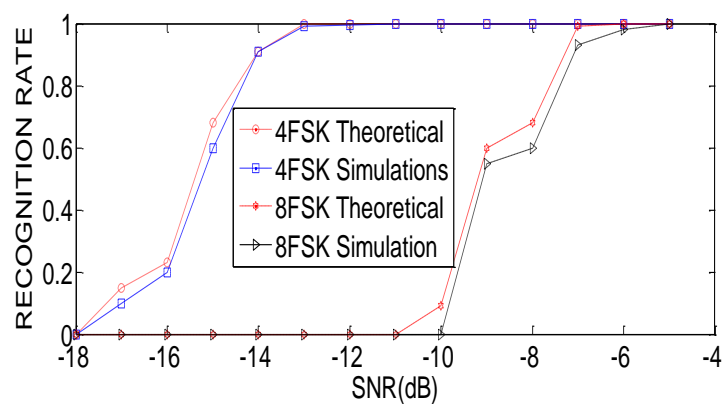

(b)

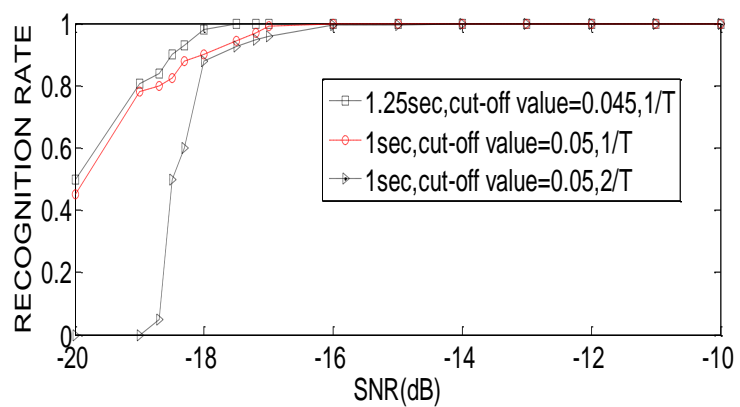

(c)

FIGURE I. THE SIMULATION FIGURE OF MODULATION CLASSIFICATION RECOGNITION

By comparing (a) and (b) in the Figure 1, the better recognition performance can be obtained for $\mathrm{AM}$ and $2 \mathrm{FSK}$ signal under the condition of lower SNR but it will be worse for high order 4FSK and SFSK signal. That is because the higher oder is, the fewer number of data series symbol observed in the receiving end in the same time slot, which makes the accuracy of estimating first-order mean value decline, results many first-order cyclic frequency are neglected under the condition of low SNR in the algorithm. So under the condition of no enough apriori information of first-order cyclic frequency, the recognition classification performance declines. In addition, the simulation result is close to the theoretical analysis. Especially when SNR is much bigger than SNR co, the classification recognition performance is better. Simulation performance of recognition classification for 2FSK signal is obtained by (b) in the figure 1 during the time slot of observation. When the time slot of observation is longer, more number of symbol series observed is obtained. Under the condition of lower cutoff value set and the related SNR co allowed to be reduced, recognition performance can also be obtained. Otherwise, with the increasing cutoff value set, needed SNR increases under the condition of same recognition performance. Meanwhile, when the time slot observed is same, the recognition performance is related to frequency offset. Smaller the frequency offset is, better recognition performance will be.

\section{CONCLUSIONS}

In this paper, the brand signal of VHF is analyzed by the algorithm of first-order cyclic mean value, which reaches the modulation classification recognition of four kinds of signal which contains AM(including ASK), FM (including FSK), MFSK and other modulation signals including DSB, SSB, PSK, MPSK, QAM, and MQAM et al. the classification recognition performance is related to the modulation order $M$, carrier phase $\theta$, time delay $t_{0}$, frequency offset $\Delta f$, SNR and the time slot observed et al. Under the condition of changless factors, when the modulation order becomes higher, frequency offset becomes smaller, time slot observed becomes smaller, the classification recognition performance of this algorithmic will decline and the result of simulation is deeply closed to the theoretical analysis. At this time, the classification recognition performance will be better and the recognition rate of four kinds of signal in the brand of VHF discussed above will reach over $98 \%$ when SNR is bigger $-6 \mathrm{~dB}$.

\section{ACKNOWLEDGEMENT}

This work was supported in part by the National Natural Science Foundation of China under Grand No. 61072070 and No. 61301179, the Doctorial Programs Foundation of the Ministry of Education under Grand No. 20110203110011, the Fundamental Research Funds of the Ministry of Education under Grand No. 72124338, the Key Programs for Natural Science Foundation of Shaanxi Province (2012JZ8002), Foundation of State Key Laboratory of Integrated Services Networks under Grand No. ISN1101002, the 111 Project under Grand No. B08038, the Projects of Science and Technology Plan Guangdong Province under Grand No. 2012B010100038.

\section{REFERENCES}

[1] Jefferson.S. \& Mengchou. Z., Real-time modulation classification based on maximum likelihood. IEEE Communications Letters, vol.35, pp. 132-140, 2008.

[2] Dennis Wong.M.L.\& Nandi.A.K., Semi-blind algorithms for automatie classifieatic for digital modulation schemes. Digital Signal Processingls, vol. 42,pp. 209-227, 2008.

[3] Oka.I.\& Fossorier.M.P.C., A General Othogonal Modulation Model for Software Radios. IEEE Transaction on Communications, vol.54,pp.712,2009

[4] Cernazanu-Glavan. C. \& Holban. S., A Model for Determining the Number of Negative Examples used in Training a MLP. Innovations in Computing Sciences and Software Engineering, vol. 36,pp. 537-542, 2010.

[5] Jian.H.\& Hua-kui.W.,MQAM Recognition based on research of constellation clustering. Wireless Communication Technology, vol.3,pp. 35-38, 2009

[6] Wei. X. J. \& Xie. H., Digital modulation recognition algorithm based on support vector machine. Electronic Design Engineering, vol.35,pp. 8991, 2011. 
[7] Wang. Y. E.\& Zhang. Q., Recognition of OFDM signals based on cyclic autocorrelation. Video Engineering, vol.68,pp. 44-48, 2012.

[8] Zhang.X.L.\& Hai L., Modulation recognition based on joint characteristics in spectrum domain. Wireless Communication Technology, vol.35,pp. 59-61, 2010.

[9] Avci. E., Hanbay. D.\& Varol. A., An expert discrete wavelet adaptive network based fuzzy inference system for digital modulation recognition. Expert Systems with Applications, vol. 3,pp. 582-589, 2007.

[10] Octavia.A.,Abdi.A.\& Wei.S., Cyclostationarity-Based Modulation Classification of Linear Digital Modulations in Flat Fading Channels. Wireless Personal Communications, vol.54,pp. 36-48, 2010.

[11] Ramkumar. B., Automatic Modulation Classification for Cognitive Radios using Cyclic Feature Detection. IEEE Circuits and Systems Magazine, vol.9, pp. 45-58, 2009.

[12] Yuan. Q., Tao. P. \& Wenbo. W., Cyclostationarity-Based Spectrum Sensing for Wideband Cognitive Radio. Proceedings of the 2009 WRI International Conference on Communications and Mobile Computing, vol.1,pp. 52-63, 2009. 